\title{
Compact Difference Scheme for Time-Fractional Fourth-Order Equation with First Dirichlet Boundary Condition
}

\author{
Mingrong Cui* \\ School of Mathematics, Shandong University, Jinan 250100, Shandong, \\ China.
}

Received 26 March 2018; Accepted (in revised version) 22 June 2018.

\begin{abstract}
The convergence of a compact finite difference scheme for one- and twodimensional time fractional fourth order equations with the first Dirichlet boundary conditions is studied. In one-dimensional case, a Hermite interpolating polynomial is used to transform the boundary conditions into the homogeneous ones. The Stephenson scheme is employed for the spatial derivatives discretisation. The approximate values of the normal derivative are obtained as a by-product of the method. For periodic problems, the stability of the method and its convergence with the accuracy $\mathscr{O}\left(\tau^{2-\alpha}\right)+\mathscr{O}\left(h^{4}\right)$ are established, with the similar error estimates for two-dimensional problems. The results of numerical experiments are consistent with the theoretical findings.
\end{abstract}

AMS subject classifications: 35R11, 65M06, 65M12, 65M15

Key words: Fractional partial differential equation, compact finite difference scheme, fourth-order equation, Stephenson scheme, stability and convergence.

\section{Introduction}

Let ${ }_{0}^{C} D_{t}^{\alpha} v, 0<\alpha<1$ be the Caputo fractional derivative of a function $v(x, t)$ [30] defined by

$$
{ }_{0}^{C} D_{t}^{\alpha} v(x, t):=\frac{1}{\Gamma(1-\alpha)} \int_{0}^{t}(t-\tau)^{-\alpha} \frac{\partial v}{\partial \tau}(x, \tau) d \tau .
$$

We consider the one-dimensional time fractional fourth-order equation

$$
{ }_{0}^{C} D_{t}^{\alpha} v(x, t)+\frac{\partial^{4} v}{\partial x^{4}}(x, t)=g(x, t), \quad x \in\left(x_{L}, x_{R}\right), \quad t \in(0, T]
$$

with the initial and boundary conditions

*Corresponding author. Email addresses: mrcui@sdu.edu. cn (Mingrong Cui) 


$$
\begin{aligned}
& v(x, 0)=v_{0}(x), \quad x \in\left[x_{L}, x_{R}\right] \\
& v\left(x_{L}, t\right)=\phi_{L}(t), \quad \frac{\partial v}{\partial x}\left(x_{L}, t\right)=\psi_{L}(t), \\
& v\left(x_{R}, t\right)=\phi_{R}(t), \quad \frac{\partial v}{\partial x}\left(x_{R}, t\right)=\psi_{R}(t) .
\end{aligned}
$$

Considering the above problem in the situation where the time variable $t$ does not appear, we obtain the biharmonic equation, which finds applications in incompressible fluid dynamics and in two-dimensional elasticity theory [2]. Numerical schemes for such problems are developed and well studied. Thus, for multi-space nonlinear parabolic partial differential equations and vibration problems, implicit difference schemes of order two in time and order four in space are, respectively, presented in [27] and [28]. It was already noted that for fourth-order diffusion equation with the second Dirichlet boundary conditions - i.e. if a second order derivative appears in the boundary conditions, the finding of numerical solutions is relatively easy. Thus writing the second order derivative as an auxiliary variable, one can split the original problem into a coupled system of two second-order equations with appropriate boundary conditions. However, the discretisation of the first Dirichlet boundary conditions requires special attention in order to match the global accuracy. As an uncoupled scheme, the Stephenson schemes of second and fourth order have been presented in [34], fourth order accurate schemes in [4] and [12], and a compact discretisation of the biharmonic problem with a fast FFT algorithm in [3].

Traditional partial differential equations contains the derivatives of integer order only. Recently, fractional differential equations attracted substantial attention because of wide applications — cf. [26,29,30]. Thus for anomalous subdiffusion equations, finite difference schemes are considered in Refs. [5,25,41,45]. Moreover, a difference scheme with spectral method [24] and fast finite difference methods $[6,37]$ are applied to space-fractional diffusion equations, to tempered fractional diffusion equations [17], to time fractional equations [22,39] and to multi-term time-fractional diffusion equations [31]. Compact finite difference schemes for subdiffusion equations are proposed and studied in $[1,8,14]$, where the error estimates $\mathscr{O}\left(\tau+h^{4}\right), \mathscr{O}\left(\tau^{2-\alpha}+h^{4}\right), \mathscr{O}\left(\tau^{2}+h^{4}\right)$ are, respectively, obtained. For one-dimensional space and time fractional Bloch-Torrey equation, the stability and convergence of a high-order difference scheme have been studied in [44] by the discrete energy method. Various high-order difference schemes for Stokes' first problem are considered for heated generalized second grade fluid with fractional derivatives [21] and for distributedorder time-fractional equations [11]. Galerkin and spectral element methods for fractional equations have been investigated in $[23,32,40]$.

The numerical solutions of fractional equations of fourth-order have been also considered - e.g. a compact algorithm for sub-diffusion equations with the first Dirichlet conditions [20]. A new variable was introduced and a high order difference scheme was developed with the convergence order $\mathscr{O}\left(\tau^{2-\alpha}+h^{4}\right)$ in $L_{2}$-norm. In addition, a local discontinuous Galerkin method for time-fractional fourth-order differential equations was studied in $[16,38]$, an implicit compact finite difference scheme for the fourth-order fractional diffusion-wave system in [19], and the hyperbolic equation describing the random vibra- 
tions of beams in $[33,43]$.

In this work, we apply the fourth order Stephenson scheme to time fractional fourth order parabolic problems with the first Dirichlet boundary conditions. This scheme is naturally suited for Dirichlet boundary conditions and, in addition to the numerical solution $\left\{\mathbf{U}^{n}\right\}$, we can simultaneously obtain the derivative $\left\{\mathbf{U}_{x}^{n}\right\}$ or partial derivatives $\left\{\mathbf{U}_{x}^{n}\right\}$ and $\left\{\mathbf{U}_{y}^{n}\right\}$ while considering one- or two-dimensional problems. The efficiency of the Stephenson scheme in solving biharmonic problems is well-known [13] and this why we use it here. Balancing the time and space errors in high-order methods one can use significantly larger space mesh size than the time step size, which allows to reduce the order of the matrices of the corresponding linear systems at each time step. This paper complements the study [20] of difference schemes for the fourth-order time fractional sub-diffusion equations.

The paper is organised as follows. In Section 2 we transform the boundary conditions into homogeneous ones, approximate the fractional time derivative by $L 1$-formula and use the one-dimensional Stephenson operator for the discretisation of spatial derivative. All this leads to a compact finite difference scheme for homogeneous problem (2.2). The stability of this scheme is considered in Section 3, while the convergence is discussed in Section 4. In the case of smooth solutions, we show that the numerical scheme under consideration has $(2-\alpha)$ order of accuracy in time and fourth order accurate in space. Section 5 deals with the extension of the method to two-dimensional problems. Numerical experiments, presented in Section 6 show that computational error can be estimated as $\mathscr{O}\left(\tau^{2-\alpha}\right)+\mathscr{O}\left(h^{4}\right)$, consistent with the theoretical analysis. Our conclusion is in Section 7.

\section{Difference Scheme}

Let us start with the transformation of the boundary conditions into homogeneous ones. Recalling the Hermite interpolation, we consider the following basis functions:

$$
\begin{aligned}
& \alpha_{L}(x)=\left[1-\frac{2\left(x-x_{L}\right)}{x_{L}-x_{R}}\right]\left(\frac{x-x_{R}}{x_{L}-x_{R}}\right)^{2}, \\
& \alpha_{R}(x)=\left[1-\frac{2\left(x-x_{R}\right)}{x_{R}-x_{L}}\right]\left(\frac{x-x_{L}}{x_{R}-x_{L}}\right)^{2}, \\
& \beta_{L}(x)=\left(x-x_{L}\right)\left(\frac{x-x_{R}}{x_{L}-x_{R}}\right)^{2}, \\
& \beta_{R}(x)=\left(x-x_{R}\right)\left(\frac{x-x_{L}}{x_{R}-x_{L}}\right)^{2} .
\end{aligned}
$$

It is easily seen that

$$
\begin{array}{llll}
\alpha_{L}\left(x_{L}\right)=1, & \frac{d \alpha_{L}}{d x}\left(x_{L}\right)=0, & \alpha_{L}\left(x_{R}\right)=0, & \frac{d \alpha_{L}}{d x}\left(x_{R}\right)=0, \\
\alpha_{R}\left(x_{L}\right)=0, & \frac{d \alpha_{R}}{d x}\left(x_{L}\right)=0, & \alpha_{R}\left(x_{R}\right)=1, & \frac{d \alpha_{R}}{d x}\left(x_{R}\right)=0, \\
\beta_{L}\left(x_{L}\right)=0, & \frac{d \beta_{L}}{d x}\left(x_{L}\right)=1, & \beta_{L}\left(x_{R}\right)=0, & \frac{d \beta_{L}}{d x}\left(x_{R}\right)=0,
\end{array}
$$




$$
\beta_{R}\left(x_{L}\right)=0, \quad \frac{d \beta_{R}}{d x}\left(x_{L}\right)=0, \quad \beta_{R}\left(x_{R}\right)=0, \quad \frac{d \beta_{R}}{d x}\left(x_{R}\right)=1 .
$$

Considering the Hermite interpolating polynomial

$$
H(x, t)=\alpha_{L}(x) \phi_{L}(t)+\alpha_{R}(x) \phi_{R}(t)+\beta_{L}(x) \psi_{L}(t)+\beta_{R}(x) \psi_{R}(t),
$$

we observe that the function $u(x, t):=v(x, t)-H(x, t)$ satisfies the boundary value problem

$$
\begin{aligned}
& { }_{0}^{C} D_{t}^{\alpha} u(x, t)+\frac{\partial^{4} u}{\partial x^{4}}(x, t)=g(x, t)-{ }_{0}^{C} D_{t}^{\alpha} H(x, t) \equiv f(x, t), \\
& x \in\left(x_{L}, x_{R}\right), \quad t \in(0, T], \\
& u(x, 0)=v_{0}(x)-H(x, 0) \equiv u_{0}(x), \quad x \in\left[x_{L}, x_{R}\right], \\
& u\left(x_{L}, t\right)=\frac{\partial u}{\partial x}\left(x_{L}, t\right)=u\left(x_{R}, t\right)=\frac{\partial u}{\partial x}\left(x_{R}, t\right)=0 .
\end{aligned}
$$

Our next task is to construct the solution of (2.2).

Remark 2.1. Since the Hermite interpolation function $H(x, t)$ is a cubic polynomial with respect to $x$, one has $\left(\partial^{4} H / \partial x^{4}\right)(x, t)=0$. That simplifies the first equation in (2.2).

Let $M$ and $N$ be positive integers. Setting $h:=\left(x_{R}-x_{L}\right) / M$ and $\tau:=T / N$, we introduce the uniform grid of mesh points $\left(x_{i}, t_{n}\right)$, where $x_{i}=x_{L}+i h, i=0,1, \cdots, M$ and $t_{n}=n \tau$, $n=0,1, \cdots, N$. Then $u\left(x_{i}, t_{n}\right)$ is the value of the exact solution at the mesh point $\left(x_{i}, t_{n}\right)$, and $U_{i}^{n}$ refer to the solution of the below difference scheme at the same mesh point.

We recall auxiliary results concerning the approximation of fractional derivatives.

Lemma 2.1 (cf. Gao \& Sun [14], Sun \& Wu [35]). If $f(t) \in C^{2}\left[0, t_{k}\right]$ and

$$
\begin{aligned}
\bar{R} f\left(t_{k}\right):= & \frac{1}{\Gamma(1-\alpha)} \int_{0}^{t_{k}} \frac{f^{\prime}(s)}{\left(t_{k}-s\right)^{\alpha}} d s-\frac{\tau^{-\alpha}}{\Gamma(2-\alpha)} \\
& \times\left[a_{0} f\left(t_{k}\right)-\sum_{j=1}^{k-1}\left(a_{k-j-1}-a_{k-j}\right) f\left(t_{i}\right)-a_{k-1} f\left(t_{0}\right)\right],
\end{aligned}
$$

then

$$
\left|\bar{R} f\left(t_{k}\right)\right| \leq \frac{1}{\Gamma(2-\alpha)}\left[\frac{1-\alpha}{12}+\frac{2^{2-\alpha}}{2-\alpha}-\left(1+2^{-\alpha}\right)\right] \max _{0 \leq t \leq t_{k}}\left|f^{\prime \prime}(t)\right| \tau^{2-\alpha},
$$

where $0<\alpha \leq 1$ and $a_{j}=(j+1)^{1-\alpha}-j^{1-\alpha}$.

Assume that $u(t) \in C^{2}\left[0, t_{n}\right]$ and consider the term

$$
D^{\alpha} u^{n}:=\frac{\tau^{-\alpha}}{\Gamma(2-\alpha)}\left[a_{0} u\left(t_{n}\right)-\sum_{l=1}^{n-1}\left(a_{n-l-1}-a_{n-l}\right) u\left(t_{l}\right)-a_{n-1} u\left(t_{0}\right)\right]
$$

with the coefficients $a_{l}=(l+1)^{1-\alpha}-l^{1-\alpha}$. It follows from Lemma 2.1 that

$$
D^{\alpha} u^{n}={ }_{0}^{C} D_{t}^{\alpha} u^{n}+\mathscr{O}\left(\tau^{2-\alpha}\right) \text { for } 0<\alpha<1 .
$$


Let $\Delta_{x} u_{i}^{n}$ and $\delta_{x}^{2} u_{i}^{n}$ be the spatial difference operators defined on the set of mesh functions $u_{i}^{n}$ by

$$
\Delta_{x} u_{i}^{n}=\frac{1}{2 h}\left(u_{i+1}^{n}-u_{i-1}^{n}\right), \quad \delta_{x}^{2} u_{i}^{n}=\frac{1}{h^{2}}\left(u_{i-1}^{n}-2 u_{i}^{n}+u_{i+1}^{n}\right) .
$$

At the interior points $x_{i}, 1 \leq i \leq M-1$ the difference scheme is constructed following the ideas of [12]. Consider an operator $\tilde{\delta}_{x}^{4}$, approximating the partial derivative $\partial^{4} / \partial x^{4}$. Let $V_{i}^{n}$ be an approximation of $(\partial u / \partial x)\left(x_{i}, t_{n}\right)$. We define the operator $\tilde{\delta}_{x}^{4}$ by

$$
\tilde{\delta}_{x}^{4} U_{i}^{n}=\frac{12}{h^{2}}\left(\Delta_{x} V_{i}^{n}-\delta_{x}^{2} U_{i}^{n}\right)
$$

This approximation of the fourth-order derivative of $u^{n}$ at $x_{i}$ is called the Stephenson's scheme [34]. Using (2.5) and Taylor series expansions, we write

$$
\tilde{\delta}_{x}^{4} u_{i}^{n}=\left.\frac{\partial^{4} u}{\partial x^{4}}\right|_{i} ^{n}+\mathscr{O}\left(h^{4}\right)
$$

We now consider an implicit compact finite difference scheme for the problem (2.2) - viz. Scheme I. Find $\left\{U_{i}^{n}\right\}, 0 \leq i \leq M, 0 \leq n \leq N$ satisfying

$$
\begin{array}{lll}
D^{\alpha} U_{i}^{n}+\tilde{\delta}_{x}^{4} U_{i}^{n}=f_{i}^{n}, & 1 \leq i \leq M-1, & 1 \leq n \leq N, \\
\frac{1}{6} V_{i-1}^{n}+\frac{2}{3} V_{i}^{n}+\frac{1}{6} V_{i+1}^{n}=\Delta_{x} U_{i}^{n}, & 1 \leq i \leq M-1, \quad 1 \leq n \leq N, \\
U_{i}^{0}=u_{0}\left(x_{i}\right), & 1 \leq i \leq M-1, & \\
U_{0}^{n}=U_{M}^{n}=V_{0}^{n}=V_{M}^{n}=0 . &
\end{array}
$$

Using the notation $\mathbf{U}^{n}:=\left(U_{1}^{n}, U_{2}^{n}, \cdots, U_{M-1}^{n}\right)^{T}$ and $\mathbf{V}^{n}:=\left(V_{1}^{n}, V_{2}^{n}, \cdots, V_{M-1}^{n}\right)^{T}$, we note that (2.6) is a coupled system - i.e. the terms $\mathbf{U}^{n}$ and $\mathbf{V}^{n}$ have to be simultaneously determined. However, we can eliminate the unknown $\mathbf{V}^{n}$, derive $\mathbf{U}^{n}$ and find $\mathbf{V}^{n}$ afterwards.

Let $\mathbf{I}$ be the identity matrix and $\mathbf{K}, \mathbf{P}, \mathbf{T}, \mathbf{S}$ be defined by

$$
\begin{aligned}
\mathbf{K} & :=\left(\begin{array}{ccccc}
0 & 1 & & & \\
-1 & 0 & 1 & & \\
& \ddots & \ddots & \ddots & \\
& & -1 & 0 & 1 \\
& & & -1 & 0
\end{array}\right)_{(M-1) \times(M-1)}, \\
\mathbf{P} & =\left(\begin{array}{ccccc}
4 & 1 & & & \\
1 & 4 & 1 & & \\
& \ddots & \ddots & \ddots & \\
& & 1 & 4 & 1 \\
& & & 1 & 4
\end{array}\right)_{(M-1) \times(M-1)} \\
\mathbf{T} & :=6 \mathbf{I}-\mathbf{P}, \quad \mathbf{S}:=\frac{6}{h^{4}}\left(3 \mathbf{K} \mathbf{P}^{-1} \mathbf{K}+2 \mathbf{T}\right) .
\end{aligned}
$$


The second equation in (2.6) can be written as

$$
\mathbf{P V}^{n}=\frac{3}{h} \mathbf{K U}^{n},
$$

and the operator $\tilde{\delta}_{x}^{4}$ has the following matrix representation:

$$
\mathbf{S u}=\frac{6}{h^{4}}\left[3 \mathbf{K P}^{-1} \mathbf{K}+2 \mathbf{T}\right] \mathbf{u} .
$$

Writing

$$
\mathbf{A}=\mathbf{S}+\frac{1}{\tau^{\alpha} \Gamma(2-\alpha)} \mathbf{I}
$$

we represent Scheme I in the form

$$
\begin{aligned}
\mathbf{A} \mathbf{U}^{1} & =\frac{1}{\tau^{\alpha} \Gamma(2-\alpha)} \mathbf{U}^{0}+\mathbf{F}^{1} \\
\mathbf{A U}^{n} & =\frac{1}{\tau^{\alpha} \Gamma(2-\alpha)} \sum_{l=1}^{n-1}\left(a_{n-l-1}-a_{n-l}\right) \mathbf{U}^{l}+\frac{1}{\tau^{\alpha} \Gamma(2-\alpha)} a_{n-1} \mathbf{U}^{0}+\mathbf{F}^{n} \\
\mathbf{U}^{0} & =\left(u_{0}\left(x_{1}\right), u_{0}\left(x_{2}\right), \cdots, u_{0}\left(x_{M-1}\right)\right)^{T}
\end{aligned}
$$

where

$$
\mathbf{F}^{n}=\left(f\left(x_{1}, t^{n}\right), f\left(x_{2}, t^{n}\right), \cdots, f\left(x_{M-1}, t^{n}\right)\right)^{T} .
$$

We point out that (2.8) contains the unknown $\left\{\mathbf{U}^{n}\right\}$ only, and $\mathbf{V}^{n}$ can be determined from (2.7) as soon as $\mathbf{U}^{n}$ have been found.

Remark 2.2. The matrix $A$ contains the factors $1 / h^{4}$ and $1 / \tau^{\alpha}$, which increase the rounding errors in the case of small $h$ and $\tau$. Therefore, in numerical experiments we multiply the corresponding equations by $\tau^{\alpha}$ to reduce the errors.

Theorem 2.1. Scheme I has a unique solution.

Proof. The coefficient matrix A in (2.8) is strictly diagonally dominant and hence invertible. Therefore, the solution of our compact finite difference scheme (2.6) exists and is unique.

\section{Stability Analysis}

For vectors $\mathbf{v}=\left(v_{0}, v_{1}, \cdots, v_{M}\right)^{T}, \mathbf{w}=\left(w_{0}, w_{1}, \cdots, w_{M}\right)^{T}$, we define the inner product and norms by

$$
(\mathbf{v}, \mathbf{w})=h \sum_{j=0}^{M} v_{j} w_{j}, \quad\|\mathbf{v}\|_{2}=(\mathbf{v}, \mathbf{v})^{1 / 2}, \quad\|\mathbf{v}\|_{\infty}=\max _{0 \leq j \leq M}\left|v_{j}\right| .
$$

In what follows, we will drop subscript 2 in the discrete $L_{2}$-norm and write it simply as $\|\cdot\|$. 
Lemma 3.1 (cf. Refs. $[3,12]$ ). The symmetric positive definite operator $\tilde{\delta}_{x}^{4}$ can be written in the form

$$
\mathbf{S}=\frac{6}{h^{4}} \mathbf{P}^{-1} \mathbf{T}^{2}+\frac{36}{h^{4}}\left(v_{1} v_{1}^{T}+v_{2} v_{2}^{T}\right)
$$

where

$$
\begin{aligned}
& v_{1}=(\alpha-\beta)^{1 / 2} \mathbf{P}^{-1}\left[\frac{\sqrt{2}}{2} e_{1}-\frac{\sqrt{2}}{2} e_{M}\right], \\
& v_{2}=(\alpha+\beta)^{1 / 2} \mathbf{P}^{-1}\left[\frac{\sqrt{2}}{2} e_{1}+\frac{\sqrt{2}}{2} e_{M}\right]
\end{aligned}
$$

with the constants

$$
\alpha=2\left(2-e_{1}^{T} \mathbf{P}^{-1} e_{1}\right), \quad \beta=2 e_{M-1}^{T} \mathbf{P}^{-1} e_{1}
$$

and the vectors $e_{1}=(1,0, \cdots, 0)^{T}, e_{M-1}=(0, \cdots, 0,1)^{T}$.

Lemma 3.2 (cf. Thomas [36]). The matrix

$$
\mathbf{T}_{\mathbf{r}}(a, b, c)=\left(\begin{array}{ccccc}
b & c & & & \\
a & b & c & & \\
& \ddots & \ddots & \ddots & \\
& & a & b & c \\
& & & a & b
\end{array}\right)_{(M-1) \times(M-1)}
$$

has the eigenvalues

$$
\lambda_{j}=b+2 c\left(\frac{a}{c}\right)^{1 / 2} \cos \frac{j \pi}{M}
$$

with the corresponding eigenvectors $\mathbf{u}_{j}=\left[u_{1}, \cdots, u_{k}, \cdots, u_{M-1}\right]^{\top}, j=1, \cdots, M-1$, where

$$
u_{k}=2\left(\frac{a}{c}\right)^{k / 2} \sin \frac{k j \pi}{M}, \quad k=1, \cdots, M-1
$$

and $\mathbf{T}$ denotes the transposition operation.

If the tridiagonal matrix $\mathbf{T}_{\mathbf{r}}(a, b, c)$ is symmetric - i.e. if $a=c$, then it is orthogonally diagonalisable and has the inverse diagonalisable by the same orthogonal matrix. Since $\mathbf{P}^{-1}$ and $\mathbf{T}^{2}$ are simultaneously diagonalisable, they commute [18]. Lemmas 3.1 and 3.2 can be used to study the properties of the matrix $\mathbf{S}$.

Lemma 3.3. The matrix $\mathbf{S}$ is symmetric positive definite and there is a positive constant $c_{0}$ such that for any vector $\mathbf{v} \neq \mathbf{0}^{T}$ the inequality

$$
(\mathbf{S v}, \mathbf{v}) \geq \frac{c_{0}}{h^{4}}\|\mathbf{v}\|^{2}
$$

holds. 
Proof. Since $\left(v_{1} v_{1}^{T}+v_{2} v_{2}^{T}\right)$ is a nonnegative definite matrix, it follows from Lemma 3.1 that

$$
(\mathbf{S v}, \mathbf{v}) \geq\left(\frac{6}{h^{4}} \mathbf{P}^{-1} \mathbf{T}^{2} \mathbf{v}, \mathbf{v}\right)
$$

Since $\mathbf{P}$ is a symmetric matrix, its eigenvectors $\left\{\varphi_{i}\right\}_{i=1}^{M-1}$ form an orthonormal matrix. By Lemma 3.2, matrices $\mathbf{T}$ and $\mathbf{P}^{-1}$ have the same set of eigenvectors. The corresponding eigenvalues, denoted by $\lambda_{i}(T)$ and $\lambda_{i}\left(P^{-1}\right)$ for $i=1, \cdots, M-1$, are positive. Since the eigenvectors $\left\{\varphi_{i}\right\}_{i=1}^{M-1}$ constitute an orthonormal basis in $\mathbb{R}^{M-1}$, any vector $\mathbf{v} \in \mathbb{R}^{M-1}$ can be represented in the form $\mathbf{v}=\sum_{i=1}^{M-1} \tilde{v}_{i} \varphi_{i}$. Then

$$
\|\mathbf{v}\|^{2}=(\mathbf{v}, \mathbf{v})=\left(\sum_{i=1}^{M-1} \tilde{v}_{i} \varphi_{i}, \sum_{j=1}^{M-1} \tilde{v}_{j} \varphi_{j}\right)=\sum_{i=1}^{M-1}\left(\tilde{v}_{i} \varphi_{i}, \tilde{v}_{i} \varphi_{i}\right)
$$

and

$$
\begin{aligned}
\left(\mathbf{P}^{-1} \mathbf{T}^{2} \mathbf{v}, \mathbf{v}\right) & =\left(\sum_{i=1}^{M-1} \lambda_{i}\left(P^{-1}\right) \lambda_{i}^{2}(T) \tilde{v}_{i} \varphi_{i}, \sum_{j=1}^{M-1} \tilde{v}_{j} \varphi_{j}\right) \\
& =\sum_{i=1}^{M-1}\left(\lambda_{i}\left(P^{-1}\right) \lambda_{i}^{2}(T) \tilde{v}_{i} \varphi_{i}, \tilde{v}_{i} \varphi_{i}\right) \\
& \geq \min _{1 \leq i \leq M-1}\left\{\lambda_{i}\left(P^{-1}\right) \lambda_{i}^{2}(T)\right\} \sum_{i=1}^{M-1}\left(\tilde{v}_{i} \varphi_{i}, \tilde{v}_{i} \varphi_{i}\right) \\
& =\min _{1 \leq i \leq M-1}\left\{\lambda_{i}\left(P^{-1}\right) \lambda_{i}^{2}(T)\right\}\|\mathbf{v}\|^{2} .
\end{aligned}
$$

Therefore, there is a constant $c_{0}>0$ independent of $h$, such that

$$
(\mathbf{S v}, \mathbf{v}) \geq \frac{c_{0}}{h^{4}}\|\mathbf{v}\|^{2}
$$

and the proof is completed.

Let $a_{l}$ be the coefficients in the Eq. (2.3). Then we have

Lemma 3.4 (cf. Refs. $[7,42]$ ). The sequence $\left\{a_{l}\right\}$ is monotonically decreasing, tends to 0 as $n$ tends to $\infty$, and satisfies the inequality

$$
(1-\alpha)(l+1)^{-\alpha}<a_{l}<(1-\alpha) l^{-\alpha} .
$$

Using Lemmas 3.3 and 3.4 , we can prove the stability of Scheme I.

Theorem 3.1. Let $\left\{\mathbf{U}^{n}\right\}(n=1, \cdots, N)$ be the solutions of Scheme I for periodic problem (1.2)(1.3), then

$$
\left\|\mathbf{U}^{n}\right\| \leq\left\|\mathbf{U}^{0}\right\|+\frac{T^{\alpha}}{1-\alpha} \Gamma(2-\alpha) \max _{1 \leq l \leq n}\left\|\mathbf{F}^{l}\right\| .
$$


Proof. Considering the inner products of $\mathbf{U}^{1}$ and the first equation in (2.8) and also $\mathbf{U}^{n}$ and the second equation in (2.8) and recalling that $a_{0}=1$, we obtain

$$
\begin{aligned}
\left(\mathbf{A U}^{n}, \mathbf{U}^{n}\right)= & \frac{1}{\tau^{\alpha} \Gamma(2-\alpha)} \sum_{l=1}^{n-1}\left(a_{n-l-1}-a_{n-l}\right)\left(\mathbf{U}^{l}, \mathbf{U}^{n}\right) \\
& +\frac{1}{\tau^{\alpha} \Gamma(2-\alpha)} a_{n-1}\left(\mathbf{U}^{0}, \mathbf{U}^{n}\right)+\left(\mathbf{F}^{n}, \mathbf{U}^{n}\right), \quad 1 \leq n \leq N .
\end{aligned}
$$

It follows from (3.1) that

$$
\begin{aligned}
\left(\mathbf{A U}^{n}, \mathbf{U}^{n}\right) & =\frac{6}{h^{4}}\left(\left(3 \mathbf{K} \mathbf{P}^{-1} \mathbf{K}+2 \mathbf{T}\right) \mathbf{U}^{n}, \mathbf{U}^{n}\right)+\frac{1}{\tau^{\alpha} \Gamma(2-\alpha)}\left(\mathbf{U}^{n}, \mathbf{U}^{n}\right) \\
& \geq\left(\frac{c_{0}}{h^{4}}+\frac{1}{\tau^{\alpha} \Gamma(2-\alpha)}\right)\left\|\mathbf{U}^{n}\right\|^{2}
\end{aligned}
$$

Therefore,

$$
\begin{aligned}
& \left|\left(\mathbf{U}^{l}, \mathbf{U}^{n}\right)\right| \leq \frac{1}{2}\left(\left\|\mathbf{U}^{l}\right\|^{2}+\left\|\mathbf{U}^{n}\right\|^{2}\right), \quad 0 \leq l \leq n-1, \\
& \left|\left(\mathbf{F}^{n}, \mathbf{U}^{n}\right)\right| \leq \frac{a_{n-1}}{2 \tau^{\alpha} \Gamma(2-\alpha)}\left\|\mathbf{U}^{n}\right\|^{2}+\frac{\tau^{\alpha} \Gamma(2-\alpha)}{2 a_{n-1}}\left\|\mathbf{F}^{n}\right\|^{2} .
\end{aligned}
$$

Using the inequalities (3.4)-(3.6) in the Eq. (3.3), we write

$$
\begin{aligned}
\left(\frac{c_{0}}{h^{4}}+\frac{1}{2 \tau^{\alpha} \Gamma(2-\alpha)}\right)\left\|\mathbf{U}^{n}\right\|^{2} \leq & \frac{1}{2 \tau^{\alpha} \Gamma(2-\alpha)} \sum_{l=1}^{n-1}\left(a_{n-l-1}-a_{n-l}\right)\left\|\mathbf{U}^{l}\right\|^{2} \\
& +\frac{1}{2 \tau^{\alpha} \Gamma(2-\alpha)} a_{n-1}\left\|\mathbf{U}^{0}\right\|^{2}+\frac{\tau^{\alpha} \Gamma(2-\alpha)}{2 a_{n-1}}\left\|\mathbf{F}^{n}\right\|^{2} .
\end{aligned}
$$

If in the left-hand side of (3.7) we replace the positive term $c_{0} / h^{4}$ by zero, it does not influence the inequality sign and (3.7) yields

$$
\left\|\mathbf{U}^{n}\right\|^{2} \leq \sum_{l=1}^{n-1}\left(a_{n-l-1}-a_{n-l}\right)\left\|\mathbf{U}^{l}\right\|^{2}+a_{n-1}\left[\left\|\mathbf{U}^{0}\right\|^{2}+\frac{\tau^{2 \alpha} \Gamma^{2}(2-\alpha)}{a_{n-1}^{2}}\left\|\mathbf{F}^{n}\right\|^{2}\right] .
$$

Following [1], we set

$$
E_{k}:=\left\|\mathbf{U}^{0}\right\|^{2}+\tau^{2 \alpha} \Gamma^{2}(2-\alpha) \max _{1 \leq l \leq k} \frac{1}{a_{l-1}^{2}}\left\|\mathbf{F}^{l}\right\|^{2},
$$

and use the method of mathematical induction to show that

$$
\left\|\mathbf{U}^{k}\right\|^{2} \leq E_{k}
$$


for all $1 \leq k \leq n$. Indeed, the inequality (3.10) is obviously valid for $k=1$. Assume that (3.10) is valid for all $k \leq n-1$. It follows from (3.9) that $E_{k}$ is a nondecreasing function of $k$. Taking into account (3.8), one obtains

$$
\begin{aligned}
\left\|\mathbf{U}^{n}\right\|^{2} & \leq \sum_{l=1}^{n-1}\left(a_{n-l-1}-a_{n-l}\right) E_{n}+a_{n-1}\left[\left\|\mathbf{U}^{0}\right\|^{2}+\frac{\tau^{2 \alpha} \Gamma^{2}(2-\alpha)}{a_{n-1}^{2}}\left\|\mathbf{F}^{n}\right\|^{2}\right] \\
& \leq \sum_{l=1}^{n-1}\left(a_{n-l-1}-a_{n-l}\right) E_{n}+a_{n-1} E_{n}=E_{n},
\end{aligned}
$$

and the inequality (3.10) is proved. Further, by Lemma 3.4,

$$
\tau^{\alpha} a_{n-1}^{-1}<\frac{T^{\alpha}}{1-\alpha}
$$

Therefore, the inequality (3.10) can be rewritten as

$$
\left\|\mathbf{U}^{n}\right\| \leq\left\|\mathbf{U}^{0}\right\|+\frac{T^{\alpha}}{1-\alpha} \Gamma(2-\alpha) \max _{1 \leq l \leq n}\left\|\mathbf{F}^{l}\right\|
$$

which finishes the proof.

\section{Error Estimates}

It is well-known that the study of numerical methods for boundary values problems is more demanding than the treatment of periodic problems. In time independent problems, the error estimates are derived by considering the matrix of biharmonic discrete operator [12]. Following this work, we transformed nonhomogeneous boundary conditions into homogeneous ones. For simplicity, here we assume that the problem under consideration is periodic, so that in spatial direction(s) the biharmonic scheme has the fourth order accuracy. On the other hand, numerical simulations show that such accuracy can be also achieved for non-periodic boundary value problems.

Discretising the homogeneous boundary value problem (2.2), we write

$$
\begin{aligned}
& D^{\alpha} \mathbf{u}^{n}+S \mathbf{u}^{n}=\mathbf{F}^{n}+\mathbf{R}^{n}, \\
& u_{0}^{n}=u_{M}^{n}=0 .
\end{aligned}
$$

The vector $\mathbf{R}^{n}$ can be represented in the form

$$
\mathbf{R}^{n}=\left(D^{\alpha} \mathbf{u}^{n}-_{0}^{C} D_{t}^{\alpha} \mathbf{u}^{n}\right)+\left(S \mathbf{u}^{n}-\frac{\partial^{4} \mathbf{u}^{n}}{\partial x^{4}}\right) \equiv \mathbf{R}_{1}^{n}+\mathbf{R}_{2}^{n},
$$

and the summands in the left-hand side of the last formula admit the estimates

$$
\left\|\mathbf{R}_{1}^{n}\right\|_{\infty}=\mathscr{O}\left(\tau^{2-\alpha}\right), \quad\left\|\mathbf{R}_{2}^{n}\right\|_{\infty}=\mathscr{O}\left(h^{4}\right),
$$

which yield

$$
\left\|\mathbf{R}^{n}\right\|_{\infty}=\mathscr{O}\left(\tau^{2-\alpha}\right)+\mathscr{O}\left(h^{4}\right) .
$$


Remark 4.1. The term $\left\|\mathbf{R}_{2}^{n}\right\|_{\infty}$ is estimated in [3], while the estimate for $\left\|\mathbf{R}_{1}^{n}\right\|_{\infty}$ follows from Lemma 2.1.

The convergence of our numerical method is described by the following theorem.

Theorem 4.1. Let $H(x, t)$ be the Hermite polynomial $(2.1), v(x, t)$ the exact solution of the periodic problem (1.2)-(1.3) and $U_{i}^{n}$ the approximate solution obtained by Scheme I. If $v(x, t) \in C_{x, t}^{8,2}\left(\left[x_{L}, x_{R}\right] \times[0, T]\right)$, then

$$
\left\|v^{n}-\left(\mathbf{U}^{n}+H^{n}\right)\right\| \leq \frac{T^{\alpha}}{1-\alpha} \Gamma(2-\alpha)\left(C_{1} \tau^{2-\alpha}+C_{2} h^{4}\right),
$$

where

$$
\begin{aligned}
& C_{1}=\frac{\sqrt{2}}{\Gamma(2-\alpha)}\left[\frac{1-\alpha}{12}+\frac{2^{2-\alpha}}{2-\alpha}-\left(1+2^{-\alpha}\right)\right] \max _{0 \leq t \leq T}\left|\frac{\partial^{2} v}{\partial t^{2}}\right|, \\
& C_{2}=\sqrt{2}\left(x_{R}-x_{L}\right) \max _{x_{L} \leq x \leq x_{R}}\left|\frac{\partial^{8} v}{\partial x^{8}}\right| .
\end{aligned}
$$

Proof. We consider the vector $\mathbf{e}^{n}=\left(e_{1}^{n}, e_{2}^{n}, \cdots, e_{M-1}^{n}\right)^{T}$ with the components $e_{i}^{n}=U_{i}^{n}-$ $\left(v\left(x_{i}, t^{n}\right)-H\left(x_{i}, t^{n}\right)\right)$, describing the error at the interior mesh points. Then

$$
\mathbf{A e}^{n}=\frac{1}{\tau^{\alpha} \Gamma(2-\alpha)} \sum_{l=1}^{n-1}\left(a_{n-l-1}-a_{n-l}\right) \mathbf{e}^{l}+\mathbf{R}^{n}, \quad 1 \leq n \leq N .
$$

It follows from (4.1) that the local truncation error $\mathbf{R}^{n}$ can be estimated as

$$
\left\|\mathbf{R}^{n}\right\| \leq C_{1} \tau^{2-\alpha}+C_{2} h^{4} .
$$

Taking into account the relation $\mathbf{e}^{0}=0$ and the estimates (4.1) and (3.2), we obtain

$$
\left\|\mathbf{e}^{n}\right\| \leq \frac{T^{\alpha}}{1-\alpha} \Gamma(2-\alpha)\left(C_{1} \tau^{2-\alpha}+C_{2} h^{4}\right),
$$

which implies the inequality (4.2).

\section{Two-Dimensional Problems}

We now turn our attention to approximate solution of two-dimensional time-fractional fourth-order problems. Our approach is based on the fourth-order compact finite difference scheme for two-dimensional biharmonic problems [3]. For simplicity, here we only consider the problems with homogeneous boundary conditions - viz.

$$
\begin{array}{lrl}
{ }_{0}^{C} D_{t} u(x, y, t)+\Delta^{2} u(x, y, t)=f(x, y, t), & (x, y) \in \Omega, & t \in(0, T], \\
u(x, y, 0)=\omega(x, y), & (x, y) \in \Omega, \\
u(x, y, t)=0, \quad \frac{\partial u}{\partial \vec{n}}(x, y, t)=0, & (x, y) \in \partial \Omega, \quad t \in(0, T],
\end{array}
$$


where $\Omega=(0, L)^{2}, \vec{n}$ is the unit outwards normal to the boundary of $\Omega$ and $\Delta^{2}$ the biharmonic operator,

$$
\Delta^{2} u(x, y, t)=\frac{\partial^{4} u}{\partial x^{4}}(x, y, t)+\frac{\partial^{4} u}{\partial y^{4}}(x, y, t)+2 \frac{\partial^{4} u}{\partial x^{2} \partial y^{2}}(x, y, t) .
$$

\subsection{Compact finite difference scheme in two dimensions}

We consider a difference scheme on a uniform mesh - viz. we set $x_{i}:=i h, y_{j}:=j h$, $0 \leq i, j \leq M$ with $h=L / M$ and $t_{n}=n \tau, 0 \leq n \leq N$ with $\tau=T / N$. The nine-point discrete Stephenson approximation of the biharmonic operator (5.2) has the form

$$
\Delta_{h}^{2} u_{i j}^{n}=\tilde{\delta}_{x}^{4} u_{i j}^{n}+\tilde{\delta}_{y}^{4} u_{i j}^{n}+2 \delta_{x}^{2} \delta_{y}^{2} u_{i j}^{n}
$$

where

$$
\begin{aligned}
\delta_{x}^{2} u_{i j}^{n} & =\frac{u_{i-1, j}^{n}-2 u_{i j}^{n}+u_{i+1, j}^{n}}{h^{2}}, \\
\delta_{y}^{2} u_{i j}^{n} & =\frac{u_{i, j-1}^{n}-2 u_{i j}^{n}+u_{i, j+1}^{n}}{h^{2}},
\end{aligned}
$$

and $\delta_{x}^{2} \delta_{y}^{2}:=\delta_{x}^{2} \circ \delta_{y}^{2}$

Let $L_{h, 0}^{2}$ be the space of sequences $\left\{u_{i j}\right\}, 0 \leq i, j \leq M$ with the zero boundary conditions $u_{i j}=0,\{i, j\} \in\{0, M\}$. If $I$ denotes the identity operator, then the Hermitian gradient $(v, w) \in\left(L_{h, 0}^{2}\right)^{2}$ is defined by

$$
\begin{aligned}
& \left(I+\frac{h^{2}}{6} \delta_{x}^{2}\right) v_{i j}^{n}=\Delta_{x} u_{i j}^{n}, \quad 1 \leq i, j \leq M-1, \\
& \left(I+\frac{h^{2}}{6} \delta_{y}^{2}\right) w_{i j}^{n}=\Delta_{y} u_{i j}^{n}, \quad 1 \leq i, j \leq M-1,
\end{aligned}
$$

and one-dimensional Stephenson operators $\tilde{\delta}_{x}^{4}$ by $\tilde{\delta}_{y}^{4}$ in the $x$ - and $y-$ directions are

$$
\begin{aligned}
& \tilde{\delta}_{x}^{4} u:=\frac{12}{h^{2}}\left(\Delta_{x} v-\delta_{x}^{2} u\right), \\
& \tilde{\delta}_{y}^{4} u:=\frac{12}{h^{2}}\left(\Delta_{y} w-\delta_{y}^{2} u\right) .
\end{aligned}
$$

It is known that the above operators approximate partial derivatives of $u$ with the fourth order accuracy, but

$$
\delta_{x}^{2} \delta_{y}^{2} u_{i j}-\left(\frac{\partial^{4} u}{\partial x^{2} \partial y^{2}}\right)_{i j}=\mathscr{O}\left(h^{2}\right) .
$$

Nevertheless, the biharmonic equation $\Delta^{2} u=f$ can be approximated with the fourth order accuracy - viz.

$$
\tilde{\Delta}_{h}^{2} u_{i j}:=\tilde{\delta}_{x}^{4}\left(I-\frac{h^{2}}{6} \delta_{y}^{2}\right) u_{i j}+\tilde{\delta}_{y}^{4}\left(I-\frac{h^{2}}{6} \delta_{x}^{2}\right) u_{i j}+2 \delta_{x}^{2} \delta_{y}^{2} u_{i j}=f_{i j}
$$


We note that the right hand side of (5.3) contains only the values $f_{i j}$, which can happen when the function $f$ is known at interior points only $[2,3]$.

Remark 5.1. The approximation properties of the operator $\tilde{\Delta}_{h}^{2}$ have been studied in [3], where Taylor series expansions are used. Here we propose another proof of the fact that the discrete difference operator $\tilde{\Delta}_{h}^{2}$ provides a fourth order approximation for the differential operator $\Delta^{2}$. Thus the mixed derivative $\partial^{4} u / \partial x^{2} \partial y^{2}$ can be approximated by the operator

$$
\left[\delta_{x}^{2}\left(I+\frac{h^{2}}{12} \delta_{x}^{2}\right)^{-1}\right] \circ\left[\delta_{y}^{2}\left(I+\frac{h^{2}}{12} \delta_{y}^{2}\right)^{-1}\right]
$$

with the fourth order accuracy. In other words, we use the usual compact finite differences $\delta_{x}^{2}\left(I+h^{2} \delta_{x}^{2} / 12\right)^{-1}$ and $\delta_{y}^{2}\left(I+h^{2} \delta_{y}^{2} / 12\right)^{-1}$ to, respectively, approximate the partial derivatives $\partial^{2} / \partial x^{2}$ and $\partial^{2} / \partial y^{2}$. Since $\delta_{x}^{2}$ and $\delta_{y}^{2}$ commute, it follows that

$$
\begin{aligned}
& {\left[\delta_{x}^{2}\left(I+\frac{h^{2}}{12} \delta_{x}^{2}\right)^{-1}\right] \circ\left[\delta_{y}^{2}\left(I+\frac{h^{2}}{12} \delta_{y}^{2}\right)^{-1}\right] } \\
= & {\left[\delta_{x}^{2}\left(I-\frac{h^{2}}{12} \delta_{x}^{2}\right)\right] \circ\left[\delta_{y}^{2}\left(I-\frac{h^{2}}{12} \delta_{y}^{2}\right)\right]+\mathscr{O}\left(h^{4}\right) I } \\
= & \delta_{x}^{2} \delta_{y}^{2}\left(I-\frac{h^{2}}{12} \delta_{x}^{2}-\frac{h^{2}}{12} \delta_{y}^{2}\right)+\mathscr{O}\left(h^{4}\right) I .
\end{aligned}
$$

Consequently, the relations

$$
\left(\delta_{x}^{2}\right)^{2}=\left(I+\frac{h^{2}}{6} \delta_{x}^{2}\right) \tilde{\delta}_{x}^{4}, \quad\left(\delta_{y}^{2}\right)^{2}=\left(I+\frac{h^{2}}{6} \delta_{y}^{2}\right) \tilde{\delta}_{y}^{4}
$$

yield

$$
\begin{aligned}
\Delta^{2} & =\frac{\partial^{4}}{\partial x^{4}}+\frac{\partial^{4}}{\partial y^{4}}+2 \frac{\partial^{4}}{\partial x^{2} \partial y^{2}} \\
& =\tilde{\delta}_{x}^{4}+\tilde{\delta}_{y}^{4}+2\left[\delta_{x}^{2}\left(I+\frac{h^{2}}{12} \delta_{x}^{2}\right)^{-1}\right] \circ\left[\delta_{y}^{2}\left(I+\frac{h^{2}}{12} \delta_{y}^{2}\right)^{-1}\right]+\mathscr{O}\left(h^{4}\right) I \\
& =\tilde{\delta}_{x}^{4}+\tilde{\delta}_{y}^{4}+2 \delta_{x}^{2} \delta_{y}^{2}\left(I-\frac{h^{2}}{12} \delta_{x}^{2}-\frac{h^{2}}{12} \delta_{y}^{2}\right)+\mathscr{O}\left(h^{4}\right) I \\
& =\tilde{\delta}_{x}^{4}+\tilde{\delta}_{y}^{4}+2 \delta_{x}^{2} \delta_{y}^{2}-\frac{h^{2}}{6}\left(\delta_{x}^{2}\right)^{2} \delta_{y}^{2}-\frac{h^{2}}{6} \delta_{x}^{2}\left(\delta_{y}^{2}\right)^{2}+\mathscr{O}\left(h^{4}\right) I \\
& =\tilde{\delta}_{x}^{4}\left(I-\frac{h^{2}}{6} \delta_{y}^{2}\right)+\tilde{\delta}_{y}^{4}\left(I-\frac{h^{2}}{12} \delta_{x}^{2}\right)+2 \delta_{x}^{2} \delta_{y}^{2}+\mathscr{O}\left(h^{4}\right) I \\
& =\tilde{\Delta}_{h}^{2}+\mathscr{O}\left(h^{4}\right) I .
\end{aligned}
$$

Using the compact scheme for steady problems and (2.4), we write the following fully discrete scheme for the problem (5.1): 
Scheme II. Find $\left\{U_{i j}^{n}\right\} \in L_{h, 0}^{2}, 0 \leq i, j \leq M, 0 \leq n \leq N$ such that

$$
\begin{array}{ll}
D^{\alpha} U_{i j}^{n}+\tilde{\Delta}_{h}^{2} U_{i j}^{n}=f_{i j}^{n}, & 1 \leq i, j \leq M-1, \quad 1 \leq n \leq N, \\
U_{i j}^{0}=\omega_{i j}, & 1 \leq i, j \leq M-1, \\
U_{i j}^{n}=0, & i, j \in\{0, M\} .
\end{array}
$$

Having determined the unknowns $U_{i j}$, we then derive $\left\{V_{i j}^{n}\right\}$ and $\left\{W_{i j}^{n}\right\}$ from the systems of equations

$$
\begin{aligned}
& \left(I+\frac{h^{2}}{6} \delta_{x}^{2}\right) V_{i j}^{n}=\Delta_{x} U_{i j}^{n}, \quad 1 \leq i, j \leq M-1, \\
& \left(I+\frac{h^{2}}{6} \delta_{y}^{2}\right) W_{i j}^{n}=\Delta_{y} U_{i j}^{n}, \quad 1 \leq i, j \leq M-1
\end{aligned}
$$

with the boundary condition $V_{i j}^{n}=W_{i j}^{n}=0$ for $\{i, j\} \in\{0, M\}$.

\subsection{Matrix form of Scheme II}

The two-dimensional finite difference operators acting on the space $L_{h, 0}^{2}$ can be associated with an $(M-1) \times(M-1), M \geq 2$ matrix operators acting on vectors $u_{i, j} \in L_{h, 0}^{2}$. We recall $[10,15]$ that the tensor (Kronecker) product $A \otimes B$ of the matrices $A \in M_{m, n}$ and $B \in M_{p, q}$ is a matrix in $M_{m \times p, n \times q}$ defined by

$$
A \otimes B=\left[\begin{array}{cccc}
a_{1,1} B & a_{1,2} B & \cdots & a_{1, n} B \\
a_{2,1} B & a_{2,2} B & \cdots & a_{2, n} B \\
\vdots & \vdots & \cdots & \vdots \\
a_{m, 1} B & a_{m, 2} B & \cdots & a_{m, n} B
\end{array}\right] .
$$

Let us write $\left\{U_{i, j}\right\}$ for the column vector

$$
\mathbf{U}=\left[U_{1,1}, \cdots, U_{1, M-1}, U_{2,1}, \cdots, U_{2, M-1}, \cdots, U_{M-1,1}, \cdots, U_{M-1, M-1}\right]^{\top} \in R^{(M-1)^{2}} .
$$

Using the so defined matrices $\mathbf{T}, \mathbf{P}$ and vectors $v_{1}, v_{2}$, we represent the bidimensional Hermitian gradient as

$$
\mathbf{V}=\frac{3}{h}\left[\mathbf{I} \otimes \mathbf{P}^{-1} \mathbf{K}\right] \mathbf{U}, \quad \mathbf{W}=\frac{3}{h}\left[\mathbf{P}^{-1} \mathbf{K} \otimes \mathbf{I}\right] \mathbf{U},
$$

the mixed derivative $\delta_{x}^{2} \delta_{y}^{2}$ as

$$
\delta_{x}^{2} \delta_{y}^{2}=\frac{1}{h^{4}} \mathbf{T} \otimes \mathbf{T}
$$

and two fourth order difference operators in two dimensions as

$$
\begin{aligned}
& \tilde{\delta}_{x}^{4}=\frac{12}{h^{2}} \mathbf{I} \otimes\left(\frac{3}{2 h^{2}} \mathbf{K} \mathbf{P}^{-1} \mathbf{K}+\frac{1}{h^{2}} \mathbf{T}\right), \\
& \tilde{\delta}_{y}^{4}=\frac{12}{h^{2}}\left(\frac{3}{2 h^{2}} \mathbf{K} \mathbf{P}^{-1} \mathbf{K}+\frac{1}{h^{2}} \mathbf{T}\right) \otimes \mathbf{I} .
\end{aligned}
$$


This differs from the notation used in [3] — cf. Remark 5.2 below. In addition, an equivalent representation of the operator $\mathbf{S}=6\left(3 \mathrm{KP}^{-1} \mathrm{~K}+2 \mathrm{~T}\right) / h^{4}$ is provided in Lemma 3.1. Thus the matrix form of the fourth order difference operator $\tilde{\Delta}_{h}^{2}$ in (5.3) is

$$
\begin{aligned}
\tilde{\Delta}_{h}^{2}= & \frac{1}{h^{4}}\left[6\left(\mathbf{I}_{M-1}+\frac{1}{6} \mathbf{T}\right) \otimes \mathbf{P}^{-1} \mathbf{T}^{2}+6 \mathbf{P}^{-1} \mathbf{T}^{2} \otimes\left(\mathbf{I}_{M-1}+\frac{1}{6} \mathbf{T}\right)+2 \mathbf{T} \otimes \mathbf{T}\right] \\
& +\frac{36}{h^{4}}\left(\mathbf{I}_{M-1}+\frac{1}{6} \mathbf{T}\right) \otimes\left[v_{1} v_{2}\right]\left[\begin{array}{l}
v_{1}^{T} \\
v_{2}^{T}
\end{array}\right]+\frac{36}{h^{4}}\left[v_{1} v_{2}\right]\left[\begin{array}{c}
v_{1}^{T} \\
v_{2}^{T}
\end{array}\right] \otimes\left(\mathbf{I}_{M-1}+\frac{1}{6} \mathbf{T}\right) .
\end{aligned}
$$

Consequently, with vectors $\left\{U_{i j}^{n}\right\} \in L_{h, 0}^{2}$, Scheme II can be written as

$$
\begin{aligned}
& \mathbf{A} \mathbf{U}^{1}=\frac{1}{\tau^{\alpha} \Gamma(2-\alpha)} \mathbf{U}^{0}+\mathbf{F}^{1}, \\
& \mathbf{A} \mathbf{U}^{n}=\frac{1}{\tau^{\alpha} \Gamma(2-\alpha)} \sum_{l=1}^{n-1}\left(a_{n-l-1}-a_{n-l}\right) \mathbf{U}^{l}+\frac{1}{\tau^{\alpha} \Gamma(2-\alpha)} a_{n-1} \mathbf{U}^{0}+\mathbf{F}^{n}, \\
& \mathbf{U}^{0}=\left(\omega_{1,1}^{0}, \cdots, \omega_{1, M-1}^{0}, \cdots, \omega_{M-1,1}^{0}, \cdots, \omega_{M-1, M-1}^{0}\right)^{T},
\end{aligned}
$$

where

$$
\mathbf{A}=\tilde{\Delta}_{h}^{2}+\frac{1}{\tau^{\alpha} \Gamma(2-\alpha)} \mathbf{I}
$$

and

$$
\mathbf{F}^{n}=\left(f_{1,1}^{n}, \cdots, f_{1, M-1}^{n}, \cdots, f_{M-1,1}^{n}, \cdots, f_{M-1, M-1}^{n}\right)^{T} .
$$

Similar to the one-dimensional case, we first derive $\left\{\mathbf{U}^{n}\right\}$ from (5.8) and then use (5.5) to determine $\left\{\mathbf{V}^{n}\right\}$ and $\left\{\mathbf{W}^{n}\right\}$.

Remark 5.2. The ordering of the column vector $\mathbf{U}$ is by rows first and then by columns, and the order of appearance of the matrices in the tensor products in (5.5)-(5.7) is different from those in [3]. Due to the symmetric form of the biharmonic operator (5.2), the fourth order difference operator $\tilde{\Delta}_{h}^{2}$ in (5.7) is unchanged - cf. [3, relation (67)]. Therefore, the final coefficient matrix $A$ in (5.8) remains the same.

Analogously to Theorem 4.1, one can show that the compact finite difference scheme (5.4) converges in discrete $L_{2}$-norm as $\mathscr{O}\left(\tau^{2-\alpha}\right)+\mathscr{O}\left(h^{4}\right)$.

Theorem 5.1. Let $u(x, y, t)$ and $\left\{U_{i j}^{n}\right\}$ be, respectively, the exact solution of the periodic problem (5.1) and the numerical solution obtained by Scheme II. Moreover, let $\mathbf{u}^{n}$ be defined on the mesh points similar to $\mathbf{U}^{n}$. If $u(x, y, t) \in C_{x, y, t}^{8,8,2}\left([0, L]^{2} \times[0, T]\right)$, then

$$
\left\|\mathbf{u}^{n}-\mathbf{U}^{n}\right\| \leq C_{1} \tau^{2-\alpha}+C_{2} h^{4}
$$

where the constants $C_{1}, C_{2}$ depend on $u, L, T$, and $\alpha$, but not on $\tau$ and $h$. 


\section{Numerical Experiments}

In this section, we consider a few numerical examples to test the convergence of our schemes. In all examples we fix $T=1$. Moreover, in the first two examples $x_{L}=0$ and $x_{R}=1$. In Example 6.3 we set $L=1$. As was already mentioned, if $\left\{\mathbf{U}^{n}\right\}$ is known, then $\left\{\mathbf{V}^{n}\right\}$ can be also found. Two-dimensional problems are handled analogously - i.e. if $\left\{\mathbf{U}^{n}\right\}$ is known, then $\left\{\mathbf{V}^{n}\right\}$ and $\left\{\mathbf{W}^{n}\right\}$ can be easily computed, which is the advantage of the Stephenson scheme. In one-dimensional setting we estimate the errors using the following discrete $L_{2}$-norm and $W^{1,2}, W^{1, \infty}$ semi-norms:

$$
\begin{aligned}
& \left\|\mathbf{e}^{N}\right\|=\left\|\mathbf{e}^{N}\right\|_{l^{2}}=\left(h \sum_{i=1}^{M-1}\left(e_{i}^{N}\right)^{2}\right)^{1 / 2}, \\
& \left|\tilde{\mathbf{e}}^{N}\right|_{1,2}=\left(h \sum_{i=1}^{M-1}\left(V_{i}^{N}-\frac{\partial u}{\partial x}\left(x_{i}, t_{N}\right)\right)^{2}\right)^{1 / 2}, \\
& \left|\tilde{\mathbf{e}}^{N}\right|_{1, \infty}=\max _{1 \leq i \leq M-1}\left|V_{i}^{N}-\frac{\partial u}{\partial x}\left(x_{i}, t_{N}\right)\right| .
\end{aligned}
$$

In two-dimensional problems, the corresponding terms are evaluated in similar way — viz.

$$
\begin{aligned}
& \left\|\mathbf{e}^{N}\right\|=\left\|\mathbf{e}^{N}\right\|_{l^{2}}=\left(h^{2} \sum_{i=1}^{M-1} \sum_{j=1}^{M-1}\left(e_{i j}^{N}\right)^{2}\right)^{1 / 2}, \\
& \left|\tilde{\mathbf{e}}^{N}\right|_{1,2}=h^{2} \sum_{i=1}^{M-1} \sum_{j=1}^{M-1}\left[\left(V_{i j}^{N}-\frac{\partial u}{\partial x}\left(x_{i}, y_{j}, t_{N}\right)\right)^{2}+\left(W_{i j}^{N}-\frac{\partial u}{\partial y}\left(x_{i}, y_{j}, t_{N}\right)\right)^{2}\right]^{1 / 2}, \\
& \left|\tilde{\mathbf{e}}^{N}\right|_{1, \infty}=\max _{1 \leq i, j \leq M-1}\left\{\left|V_{i j}^{N}-\frac{\partial u}{\partial x}\left(x_{i}, y_{j}, t_{N}\right)\right|,\left|W_{i j}^{N}-\frac{\partial u}{\partial y}\left(x_{i}, y_{j}, t_{N}\right)\right|\right\} .
\end{aligned}
$$

The approximate discrete $W^{1,2}, W^{1, \infty}$ semi-norms estimate the difference between the gradient of the exact solutions and the corresponding numerical approximations $\left\{\mathbf{V}^{n}\right\},\left\{\mathbf{W}^{n}\right\}$. Recalling that $\left\|\mathbf{e}^{N}\right\|$ depends on $\tau$ and $h$, we write it as $\left\|\mathbf{e}\left(\tau, h, t^{N}\right)\right\|$ for clarity. Since $N$ is the integer part $[1 / \tau]$ of $1 / \tau$, we have $N \tau \approx 1$. To test the numerical convergence, we will follow the approach of [9]. Noting the theoretical estimate (5.9), we can expect that the replacing $\tau$ by $\tau / 2^{4 /(2-\alpha)}$ and $h$ by $h / 2$, makes the numerical error estimate 16 times smaller since

$$
\begin{aligned}
\left\|\mathbf{e}\left(\tau / 2^{4 /(2-\alpha)}, h / 2, t^{\tilde{N}}\right)\right\| & \approx C_{1}\left(\tau / 2^{4 /(2-\alpha)}\right)^{2-\alpha}+C_{2}(h / 2)^{4} \\
& =\frac{1}{16}\left(C_{1} \tau^{2-\alpha}+C_{2} h^{4}\right) \approx \frac{1}{16}\left\|\mathbf{e}\left(\tau, h, t^{N}\right)\right\|
\end{aligned}
$$

for $N$ and $\tilde{N}$ such that $N \tau \approx \tilde{N} \tau / 2^{4 /(2-\alpha)} \approx 1$. In addition, one of the parameters $\tau$ or $h$ can be chosen sufficiently small to ignore its influence on the error estimate. It allows to test spatial and temporal convergence rates separately by using the relation

$$
\left\|\mathbf{e}\left(\tau, h, t^{N}\right)\right\|=\mathscr{O}\left(\tau^{2-\alpha}\right)+\mathscr{O}\left(h^{4}\right) \leq C_{1} \tau^{2-\alpha}+C_{2} h^{4} .
$$


Of course, such a test depends on the assumption that the lower and upper bounds for the ratio $C_{1} / C_{2}$ are known in advance, so that we can decide which parameter shall be small. Here we combine these two tests into one by an appropriate choice of mesh sizes. Consequently, in the numerical tests the experimental convergence order $r=r(\tau, h)$ is calculated as

$$
r(\tau, h)=\log _{2}\left(\left\|\mathbf{e}\left(\tau, h, t^{N_{1}}\right)\right\|_{*} /\left\|\mathbf{e}\left(\tau / 2^{4 /(2-\alpha)}, h / 2, t^{N_{2}}\right)\right\|_{*}\right),
$$

where $N_{1}=[1 / \tau]$ and $N_{2}=\left[2^{4 /(2-\alpha)} / \tau\right]$. As already mentioned, we expect that $r=$ $r(\tau, h) \approx \log _{2} 16=4$. Let us also note that in the numerical simulations, we consider the cases $\alpha=0.25, \alpha=0.5$ and $\alpha=0.75$.

Example 6.1 (Homogeneous boundary conditions). We consider the problem (2.2), which has the solution $u(x, t)=t^{3} \sin ^{2}(\pi x)$. In this case,

$$
u(0, t)=\frac{\partial u}{\partial x}(1, t)=0
$$

and

$$
f(x, t)=\frac{6 t^{3-\alpha}}{\Gamma(4-\alpha)} \sin ^{2}(\pi x)-8 \pi^{4} t^{3} \cos (2 \pi x) .
$$

The errors of the compact scheme and the experimental convergence order are shown in Tables 1-3. We note that they are consistent with theoretical results.

Example 6.2 (Nonhomogeneous boundary conditions). We consider problem (1.2)-(1.3), which has the solution $v(x, t)=t^{3} e^{x}$. In this case,

$$
g(x, t)=\left(\frac{6 t^{3-\alpha}}{\Gamma(4-\alpha)}+t^{3}\right) e^{x},
$$

and the Hermite interpolation function has the form

$$
H(x, t)=\left[(1+3 x)(x-1)^{2}+(2-x) x^{2} e\right] t^{3} .
$$

The corresponding errors of the compact scheme are presented in Tables 4-6 and the accuracy meets our expectations.

Example 6.3 (Two-dimensional problem. Homogeneous boundary conditions). We consider the problem (5.1), which has the solution $u(x, y, t)=t^{3} \sin ^{2}(\pi x) \sin ^{2}(\pi y)$. In this case,

$$
\begin{aligned}
f(x, y, t)= & \frac{3 t^{3-\alpha}}{2 \Gamma(4-\alpha)}(1-\cos (2 \pi x))(1-\cos (2 \pi y)) \\
& +4 \pi^{4} t^{3}(4 \cos (2 \pi x) \cos (2 \pi y)-\cos (2 \pi x)-\cos (2 \pi y)) .
\end{aligned}
$$

The corresponding errors of the compact scheme are presented in Tables 7-9 and they are consistent with the theoretical estimates. 
Table 1: Error and experiment order of convergence of Scheme I for $\alpha=0.25$ (Example 6.1).

\begin{tabular}{||lccccccccc||}
\hline$M$ & $N$ & $\left\|\mathrm{e}^{N}\right\|_{\infty}$ & order & $\left\|\mathrm{e}^{N}\right\|$ & order & $\left|\tilde{\mathrm{e}}^{N}\right|_{1, \infty}$ & order & $\left|\tilde{\mathrm{e}}^{N}\right|_{1,2}$ & order \\
\hline 5 & 5 & 0.0045 & - & 0.0031 & - & 0.0351 & - & 0.0261 & - \\
10 & 24 & $2.3045 \mathrm{e}-4$ & 4.2874 & $1.4118 \mathrm{e}-4$ & 4.4567 & 0.0019 & 4.2074 & 0.0014 & 4.2206 \\
20 & 119 & $1.4176 \mathrm{e}-5$ & 4.0229 & $8.6857 \mathrm{e}-6$ & 4.0227 & $1.2804 \mathrm{e}-4$ & 3.8913 & $9.0523 \mathrm{e}-5$ & 3.9510 \\
40 & 580 & $8.7137 \mathrm{e}-7$ & 4.0240 & $5.3391 \mathrm{e}-7$ & 4.0240 & $7.9399 \mathrm{e}-6$ & 4.0113 & $5.6135 \mathrm{e}-6$ & 4.0113 \\
80 & 2826 & $5.4190 \mathrm{e}-8$ & 4.0072 & $3.3205 \mathrm{e}-8$ & 4.0113 & $4.9415 \mathrm{e}-7$ & 4.0061 & $3.4935 \mathrm{e}-7$ & 4.0062 \\
\hline
\end{tabular}

Table 2: Error and experiment order of convergence of Scheme I for $\alpha=0.5$ (Example 6.1).

\begin{tabular}{||lccccccccc||}
\hline$M$ & $N$ & $\left\|\mathrm{e}^{N}\right\|_{\infty}$ & order & $\left\|\mathrm{e}^{N}\right\|$ & order & $\left|\tilde{\mathbf{e}}^{N}\right|_{1, \infty}$ & order & $\left|\tilde{\mathrm{e}}^{N}\right|_{1,2}$ & order \\
\hline 5 & 5 & 0.0047 & - & 0.0032 & - & 0.0347 & - & 0.0258 & - \\
10 & 32 & $2.5662 \mathrm{e}-4$ & 4.1950 & $1.5736 \mathrm{e}-4$ & 4.3459 & 0.0020 & 4.1169 & 0.0015 & 4.1043 \\
20 & 202 & $1.4814 \mathrm{e}-5$ & 4.1146 & $9.0862 \mathrm{e}-6$ & 4.1142 & $1.2662 \mathrm{e}-4$ & 3.9814 & $8.9497 \mathrm{e}-5$ & 4.0670 \\
40 & 1280 & $9.0650 \mathrm{e}-7$ & 4.0305 & $5.5603 \mathrm{e}-7$ & 4.0304 & $7.8144 \mathrm{e}-6$ & 4.0182 & $5.5231 \mathrm{e}-6$ & 4.0183 \\
80 & 8127 & $5.6431 \mathrm{e}-8$ & 4.0057 & $3.4614 \mathrm{e}-8$ & 4.0057 & $4.8753 \mathrm{e}-7$ & 4.0026 & $3.4457 \mathrm{e}-7$ & 4.0026 \\
\hline
\end{tabular}

Table 3: Error and experiment order of convergence of Scheme I for $\alpha=0.75$ (Example 6.1).

\begin{tabular}{||lccccccccc||}
\hline$M$ & $N$ & $\left\|\mathrm{e}^{N}\right\|_{\infty}$ & order & $\left\|\mathrm{e}^{N}\right\|$ & order & $\left|\tilde{\mathrm{e}}^{N}\right|_{1, \infty}$ & order & $\left|\tilde{\mathrm{e}}^{N}\right|_{1,2}$ & order \\
\hline 5 & 5 & 0.0049 & - & 0.0033 & - & 0.0339 & - & 0.0252 & - \\
10 & 46 & $2.7107 \mathrm{e}-4$ & 4.1760 & $1.6653 \mathrm{e}-4$ & 4.3086 & 0.0019 & 4.1572 & 0.0014 & 4.1699 \\
20 & 422 & $1.5904 \mathrm{e}-5$ & 4.0912 & $9.7732 \mathrm{e}-6$ & 4.0908 & $1.2196 \mathrm{e}-4$ & 3.9615 & $8.6144 \mathrm{e}-5$ & 4.0225 \\
40 & 3880 & $9.8077 \mathrm{e}-7$ & 4.0193 & $6.0272 \mathrm{e}-7$ & 4.0193 & $7.5862 \mathrm{e}-6$ & 4.0069 & $5.3584 \mathrm{e}-6$ & 4.0069 \\
80 & 35658 & $6.1088 \mathrm{e}-8$ & 4.0050 & $3.7542 \mathrm{e}-8$ & 4.0049 & $4.7347 \mathrm{e}-7$ & 4.0020 & $3.3442 \mathrm{e}-7$ & 4.0021 \\
\hline
\end{tabular}

Table 4: Error and experiment order of convergence of Scheme I for $\alpha=0.25$ (Example 6.2).

\begin{tabular}{||lccccccccc||}
\hline$M$ & $N$ & $\left\|\mathrm{e}^{N}\right\|_{\infty}$ & order & $\left\|\mathrm{e}^{N}\right\|$ & order & $\left|\tilde{\mathbf{e}}^{N}\right|_{1, \infty}$ & order & $\left|\tilde{\mathrm{e}}^{N}\right|_{1,2}$ & order \\
\hline 5 & 5 & $2.6849 \mathrm{e}-6$ & - & $1.6494 \mathrm{e}-6$ & - & $2.2274 \mathrm{e}-5$ & - & $1.6646 \mathrm{e}-5$ & - \\
10 & 24 & $1.6341 \mathrm{e}-7$ & 4.0383 & $9.7432 \mathrm{e}-8$ & 4.0814 & $1.3638 \mathrm{e}-6$ & 4.0297 & $9.9709 \mathrm{e}-7$ & 4.0613 \\
20 & 119 & $1.0842 \mathrm{e}-8$ & 3.9138 & $6.3566 \mathrm{e}-9$ & 3.9381 & $8.9968 \mathrm{e}-8$ & 3.9221 & $6.5600 \mathrm{e}-8$ & 3.9260 \\
40 & 580 & $6.7303 \mathrm{e}-10$ & 4.0098 & $3.9382 \mathrm{e}-10$ & 4.0126 & $5.6259 \mathrm{e}-9$ & 3.9993 & $4.0963 \mathrm{e}-9$ & 4.0013 \\
80 & 2826 & $4.1730 \mathrm{e}-11$ & 4.0115 & $2.4366 \mathrm{e}-11$ & 4.0146 & $3.5128 \mathrm{e}-10$ & 4.0014 & $2.5551 \mathrm{e}-10$ & 4.0029 \\
\hline
\end{tabular}

Table 5: Error and experiment order of convergence of Scheme I for $\alpha=0.5$ (Example 6.2).

\begin{tabular}{||lccccccccc||}
\hline$M$ & $N$ & $\left\|\mathrm{e}^{N}\right\|_{\infty}$ & order & $\left\|\mathrm{e}^{N}\right\|$ & order & $\left|\tilde{\mathrm{e}}^{N}\right|_{1, \infty}$ & order & $\left|\tilde{\mathrm{e}}^{N}\right|_{1,2}$ & order \\
\hline 5 & 5 & $2.4385 \mathrm{e}-6$ & - & $1.3914 \mathrm{e}-6$ & - & $2.3271 \mathrm{e}-5$ & - & $1.6740 \mathrm{e}-5$ & - \\
10 & 32 & $1.5356 \mathrm{e}-7$ & 3.9891 & $8.7746 \mathrm{e}-8$ & 3.9871 & $1.5077 \mathrm{e}-6$ & 3.9481 & $1.0782 \mathrm{e}-6$ & 3.9566 \\
20 & 202 & $9.3615 \mathrm{e}-9$ & 4.0359 & $5.3191 \mathrm{e}-9$ & 4.0441 & $9.3060 \mathrm{e}-8$ & 4.0180 & $6.6349 \mathrm{e}-8$ & 4.0224 \\
40 & 1280 & $5.8366 \mathrm{e}-10$ & 4.0035 & $3.2869 \mathrm{e}-10$ & 4.0164 & $5.7912 \mathrm{e}-9$ & 4.0062 & $4.1241 \mathrm{e}-9$ & 4.0079 \\
80 & 8127 & $3.6398 \mathrm{e}-11$ & 4.0032 & $2.0487 \mathrm{e}-11$ & 4.0039 & $3.6238 \mathrm{e}-10$ & 3.9983 & $2.5778 \mathrm{e}-10$ & 3.9999 \\
\hline
\end{tabular}


Table 6: Error and experiment order of convergence of Scheme I for $\alpha=0.75$ (Example 6.2).

\begin{tabular}{||lccccccccc||}
\hline$M$ & $N$ & $\left\|\mathbf{e}^{N}\right\|_{\infty}$ & order & $\left\|\mathbf{e}^{N}\right\|$ & order & $\left|\tilde{\mathbf{e}}^{N}\right|_{1, \infty}$ & order & $\left|\tilde{\mathbf{e}}^{N}\right|_{1,2}$ & order \\
\hline 5 & 5 & $1.9062 \mathrm{e}-6$ & - & $1.1404 \mathrm{e}-6$ & - & $2.5427 \mathrm{e}-5$ & - & $1.7309 \mathrm{e}-5$ & - \\
10 & 46 & $1.1486 \mathrm{e}-7$ & 4.0528 & $7.4062 \mathrm{e}-8$ & 3.9447 & $1.6283 \mathrm{e}-6$ & 3.9649 & $1.0998 \mathrm{e}-6$ & 3.9762 \\
20 & 422 & $7.1276 \mathrm{e}-9$ & 4.0103 & $4.6332 \mathrm{e}-9$ & 3.9987 & $1.0326 \mathrm{e}-7$ & 3.9790 & $6.8573 \mathrm{e}-8$ & 4.0035 \\
40 & 3880 & $4.5173 \mathrm{e}-10$ & 3.9799 & $2.9021 \mathrm{e}-10$ & 3.9968 & $6.4670 \mathrm{e}-9$ & 3.9970 & $4.2940 \mathrm{e}-9$ & 3.9972 \\
80 & 35658 & $2.8208 \mathrm{e}-11$ & 4.0013 & $1.8147 \mathrm{e}-11$ & 3.9993 & $4.0454 \mathrm{e}-10$ & 3.9987 & $2.6849 \mathrm{e}-10$ & 3.9994 \\
\hline
\end{tabular}

Table 7: Error and experiment order of convergence of Scheme II for $\alpha=0.25$ (Example 6.3).

\begin{tabular}{||lccccccccc||}
\hline$M$ & $N$ & $\left\|\mathbf{e}^{N}\right\|_{\infty}$ & order & $\left\|\mathbf{e}^{N}\right\|$ & order & $\left|\tilde{\mathbf{e}}^{N}\right|_{1, \infty}$ & order & $\left|\tilde{\mathbf{e}}^{N}\right|_{1,2}$ & order \\
\hline 5 & 5 & 0.0041 & - & 0.0019 & - & 0.0319 & - & 0.0227 & - \\
10 & 24 & $2.2801 \mathrm{e}-4$ & 4.1685 & $8.5543 \mathrm{e}-5$ & 4.4732 & 0.0019 & 4.0695 & 0.0012 & 4.2416 \\
20 & 119 & $1.3997 \mathrm{e}-5$ & 4.0259 & $5.2517 \mathrm{e}-6$ & 4.0258 & $1.2859 \mathrm{e}-4$ & 3.8851 & $7.8731 \mathrm{e}-5$ & 3.9300 \\
40 & 580 & $8.5916 \mathrm{e}-7$ & 4.0260 & $3.2238 \mathrm{e}-7$ & 4.0260 & $7.9775 \mathrm{e}-6$ & 4.0107 & $4.8842 \mathrm{e}-6$ & 4.0107 \\
\hline
\end{tabular}

Table 8: Error and experiment order of convergence of Scheme II for $\alpha=0.5$ (Example 6.3).

\begin{tabular}{||lccccccccc||}
\hline$M$ & $N$ & $\left\|\mathbf{e}^{N}\right\|_{\infty}$ & order & $\left\|\mathbf{e}^{N}\right\|$ & order & $\left|\tilde{\mathbf{e}}^{N}\right|_{1, \infty}$ & order & $\left|\tilde{\mathrm{e}}^{N}\right|_{1,2}$ & order \\
\hline 5 & 5 & 0.0041 & - & 0.0019 & - & 0.0317 & - & 0.0226 & - \\
10 & 32 & $2.4817 \mathrm{e}-4$ & 4.0462 & $9.3199 \mathrm{e}-5$ & 4.3495 & 0.0020 & 3.9864 & 0.0013 & 4.1197 \\
20 & 202 & $1.4259 \mathrm{e}-5$ & 4.1214 & $5.3562 \mathrm{e}-6$ & 4.1210 & $1.2833 \mathrm{e}-4$ & 3.9621 & $7.8544 \mathrm{e}-5$ & 4.0489 \\
40 & 1280 & $8.7118 \mathrm{e}-7$ & 4.0328 & $3.2727 \mathrm{e}-7$ & 4.0327 & $7.9231 \mathrm{e}-6$ & 4.0176 & $4.8491 \mathrm{e}-6$ & 4.0177 \\
\hline
\end{tabular}

Table 9: Error and experiment order of convergence of Scheme II for $\alpha=0.75$ (Example 6.3).

\begin{tabular}{||lccccccccc||}
\hline$M$ & $N$ & $\left\|\mathrm{e}^{N}\right\|_{\infty}$ & order & $\left\|\mathrm{e}^{N}\right\|$ & order & $\left|\tilde{\mathrm{e}}^{N}\right|_{1, \infty}$ & order & $\left|\tilde{\mathrm{e}}^{N}\right|_{1,2}$ & order \\
\hline 5 & 5 & 0.0042 & - & 0.0019 & - & 0.0314 & - & 0.0224 & - \\
10 & 46 & $2.5040 \mathrm{e}-4$ & 4.0681 & $9.4236 \mathrm{e}-5$ & 4.3336 & 0.0020 & 3.9727 & 0.0013 & 4.1069 \\
20 & 422 & $1.4592 \mathrm{e}-5$ & 4.1010 & $5.4934 \mathrm{e}-6$ & 4.1005 & $1.2600 \mathrm{e}-4$ & 3.9885 & $7.7055 \mathrm{e}-5$ & 4.0765 \\
40 & 3880 & $8.9846 \mathrm{e}-7$ & 4.0216 & $3.3826 \mathrm{e}-7$ & 4.0215 & $7.8396 \mathrm{e}-6$ & 4.0065 & $4.7943 \mathrm{e}-6$ & 4.0065 \\
\hline
\end{tabular}

\section{Conclusion}

We studied the convergence of a compact finite difference scheme for one- and twodimensional time fractional fourth order equations with the first Dirichlet boundary conditions. In one-dimensional case, we used a Hermitian interpolation function to transform it into a problem with homogeneous boundary conditions. The Stephenson scheme is used for spatial derivatives discretisation. As a by-product of the method, the approximate values of normal derivatives are obtained. For periodic problems, the scheme is proved to be stable and convergent with the accuracy $\mathscr{O}\left(\tau^{2-\alpha}\right)+\mathscr{O}\left(h^{4}\right)$. In two-dimensional problems, the error estimates are similar. The results of numerical experiments are consistent with the theoretical analysis. 


\section{Acknowledgments}

The author thanks Professors Victor Didenko, Tao Zhou and the anonymous referees for valuable comments and suggestions.

This work was partially supported by the Natural Science Foundation of the Shandong Province (Grant No. ZR2014AM013), by the NSFC (Grant No. 11471194) and by the NSAF (Grant No. U1430101). Some results of this work have been presented on the workshop "Numerical Solution of Fractional Differential Equations" held in the South-East University, Nanjing in October 2017. The author also thanks Prof. Zhi-Zhong Sun for his kind invitation.

\section{References}

[1] A.A. Alikhanov, A new difference scheme for the time fractional diffusion equation, J. Comput. Phys. 280, 424-438 (2015).

[2] M. Ben-Artzi, J.-P. Croisille and D. Fishelov, Convergence of a compact scheme for the pure streamfunction formulation of the unsteady Navier-Stokes system, SIAM J. Numer. Anal. 44, 1997-2024 (2006).

[3] M. Ben-Artzi, J.-P. Croisille and D. Fishelov, A fast direct solver for the biharmonic problem in a rectangular grid, SIAM J. Sci. Comput. 31, 303-333 (2008).

[4] D. Bialecki, A fourth order finite difference method for the Dirichlet biharmonic problem, Numer. Algor. 61, 351-375 (2012).

[5] C.-M. Chen, F. Liu, I. Turner and V. Anh, A Fourier method for the fractional diffusion equation describing sub-diffusion, J. Comput. Phys. 227, 886-897 (2007).

[6] S. Chen, F. Liu, X. Jiang, I. Turner and K. Burrage, Fast finite difference approximation for identifying parameters in a two-dimensional space-fractional nonlocal model with variable diffusivity coefficients, SIAM J. Numer. Anal. 54, 606-624 (2016).

[7] S. Chen, F. Liu, P. Zhuang and V. Anh, Finite difference approximations for the fractional FokkerPlanck equation, Appl. Math. Model. 33, 256-273 (2009).

[8] M.R. Cui, Compact finite difference method for the fractional diffusion equation, J. Comput. Phys. 228, 7792-7804 (2009).

[9] M.R. Cui, Convergence analysis of high-order compact alternating direction implicit schemes for the two-dimensional time fractional diffusion equation, Numer. Algor. 62, 383-409 (2013).

[10] J.W. Demmel, Applied Numerical Linear Algebra, SIAM (1997).

[11] R. Du, Z.-P. Hao and Z.-Z. Sun, Lubich second-order methods for distributed-order time-fractional differential equations with smooth solutions, East Asian J. Appl. Math. 6, 131-151 (2018).

[12] D. Fishelov, M. Ben-Artzi and J.-P. Croisille, Recent advances in the study of a fourth-order compact scheme for the one-dimensional biharmonic equations, J. Sci. Comput. 53, 55-79 (2012).

[13] M. Fishelov, J.-P. Croisille and D. Fishelov, Navier-Stokes Equations in Planar Domains, Imperial College Press (2013).

[14] G.-H. Gao and Z.-Z. Sun, A compact finite difference scheme for the fractional sub-diffusion equations, J. Comput. Phys. 230, 586-595 (2011).

[15] G.H. Golub and C.F. Van Loan, Matrix Computations, 3rd ed, John Hopkins University Press (1996).

[16] L. Guo, Z.B. Wang and S. Vong, Fully discrete local discontinuous Galerkin methods for some time-fractional fourth-order problems, Inter. J. Comput. Math. 93, 1665-1682 (2016). 
[17] X. Guo, Y.T. Li and H. Wang, A fast finite difference method for tempered fractional diffusion equations, Commun. Comput. Phys. 24, 531-556 (2018).

[18] R.A. Horn and C.R. Johnson, Matrix Analysis, Cambridge University Press (1985).

[19] X.L. Hu and L.M. Zhang, A new implicit compact difference scheme for the fourth-order fractional diffusion-wave system, Inter. J. Comput. Math. 91, 2215-2231 (2014).

[20] C.-C. Ji, Z.-Z. Sun and Z.-P. Hao, Numerical algorithms with high spatial accuracy for the fourthorder fractional sub-diffusion equations with the first Dirichlet boundary conditions, J. Sci. Comput. 66, 1148-1174 (2016).

[21] C.-C. Ji and Z.Z. Sun, An unconditionally stable and high-order convergent difference scheme for Stokes' first problem for a heated generalized second grade fluid with fractional derivative, Numer. Math. Theor. Meth. Appl. 10, 597-613 (2017).

[22] S. Jiang, J. Zhang, Q. Zhang and Z. Zhang, Fast evaluation of the Caputo fractional derivative and its applications to fractional diffusion equations, Commun. Comput. Phys. 21, 650-678 (2017).

[23] D.F. Li, H.-L. Liao, W.W. Sun, J. L. Wang and J. W. Zhang, Analysis of L1-Galerkin FEMs for time-fractional nonlinear parabolic problems, Commun. Comput. Phys. 24, 86-103 (2018).

[24] Y. Lin and C. Xu, Finite difference/spectral approximations for the time-fractional diffusion equation, J. Comput. Phys. 225, 1533-1552 (2007).

[25] F.W. Liu, P.H. Zhuang and Q.X. Liu, Numerical Methods for Fractional Partial Differential Equations with Applications (In Chinese), Information and Computational Sciences Series, vol 74, Science Press (2016).

[26] R. Metzler and J. Klafter, The random walk's guide to anomalous diffusion, a fractional dynamics approach, Phys. Rep. 339, 1-77 (2000).

[27] R.K. Mohanty and D. Kaur, High accuracy compact operator methods for two-dimensional fourth order nonlinear parabolic partial differential equations, Comput. Methods Appl. Math. 17, 617641 (2017).

[28] R.K. Mohanty and D. Mohanty, Unconditionally stable high accuracy compact difference schemes for multi-space dimensional vibration problems with simply supported boundary conditions, Appl. Math. Model. 55, 281-298 (2018).

[29] K.B. Oldham and J. Spanier, The Fractional Calculus, Theory and Applications of Differentiation and Integration of Arbitray Order, Academic Press (1974).

[30] I. Podlubny, Fractional Differential Equations, Academic Press (1999).

[31] J.C. Ren and Z.-Z. Sun, Efficient and stable numerical methods for the multi-term time fractional subdiffusion equations, East Asian J. Appl. Math. 4, 242-266 (2014).

[32] C.T. Sheng and J. Shen, A Hybrid spectral element method for fractional two-point boundary value problems, Numer. Math. Theor. Meth. Appl. 10, 437-464 (2017).

[33] P.D. Spanos and G. Malara, Nonlinear random vibrations of beams with fractional derivative elements, J. Eng. Mech. 140, 04014069 (2014).

[34] J.W. Stephenson, Single cell discretizations of order two and four for biharmonic problems, J. Comput. Phys. 55, 65-80 (1984).

[35] Z.-Z. Sun, X.N. Wu, A fully discrete difference scheme for a diffusion-wave system, Appl. Numer. Math. 56, 193-209 (2006).

[36] J.W. Thomas, Numerical Partial Differential Equations, Finite Difference Methods, Texts in Applied Mathematics, 22, Springer (1995).

[37] H. Wang, K. Wang and T. Sircar, A direct $O\left(N \log ^{2} N\right)$ finite difference method for fractional diffusion equations, J. Comput. Phys. 229, 8095-8104 (2010).

[38] L.L. Wei and Y. N. He, Analysis of a fully discrete local discontinuous Galerkin method for timefractional fourth-order problems, Appl. Math. Model. 38, 1511-1522 (2014). 
[39] Y.G. Yan, Z.-Z. Sun and J.W. Zhang, Fast evaluation of the Caputo fractional derivative and its applications to fractional diffusion equations: A second-order scheme, Commun. Comput. Phys. 22, 1028-1048 (2017).

[40] Y.B. Yang and H.P. Ma, The Legendre Galerkin-Chebyshev collocation method for space fractional Burgers-like equations, Numer. Math. Theor. Meth. Appl. 11, 338-353 (2018).

[41] S.B. Yuste and L. Acedo, An explicit finite difference method and a new von Neumann-type stability analysis for fractional diffusion equations, SIAM J. Numer. Anal. 42, 1862-1874 (2005).

[42] Y.-N. Zhang and Z.-Z. Sun, Alternating direction implicit schemes for the two-dimensional fractional sub-diffusion equation, J. Comput. Phys. 230, 8713-8728 (2011).

[43] S.Y. Zhu, C.B. Cai and P.D. Spanos, A nonlinear and fractional derivative viscoelastic model for rail pads in the dynamic analysis of coupled vehicle-slab track systems, J. Sound Vibra. 335, 304-320 (2015).

[44] Y. Zhu and Z.-Z. Sun, A high-order difference scheme for the space and time fractional BlochTorrey equation, Comput. Methods Appl. Math. 18, 147-164 (2018).

[45] P. Zhuang, F. Liu, V. Anh and I. Turner, New solution and analytical techniques of the implicit numerical method for the anomalous subdiffusion equation, SIAM J. Numer. Anal. 46, 10791095 (2008). 